rem that $(p / q$ being irreducible)

$$
p=p_{n}+\epsilon p_{n-1}, \quad q=q_{n}+\epsilon q_{n-1}, \quad \epsilon= \pm 1,
$$

for the convergents to $x$ are all $[e / o]$ or $[o / e]$. Write $X=\left[a_{n+1}\right.$, $\left.a_{n+2}, \cdots\right], Y=\left[a_{n}, a_{n-1}, \cdots, a_{2}\right]$. Then if $n \geqq 2$,

$$
\theta=\frac{(Y+\epsilon)(X-\epsilon)}{X Y+1}=1-\frac{2-\epsilon(X-Y)}{X Y+1}>1-\frac{2+X+Y}{X Y+1},
$$

$$
\begin{aligned}
X Y+1-E(2+X+Y) & =(X-E)(Y-E)-E^{2}-2 E+1 \\
> & (E+1)^{2}-E^{2}-2 E+1>0, \\
& \theta>1-1 / E .
\end{aligned}
$$

If $n=1$, then $p=p_{1}+1, q=q_{1}=1, \theta=1-\left[0, a_{2}, \cdots\right]>1-1 / E$.

Raffles College, Singapore

\title{
MEASURABILITY AND DISTRIBUTIVITY IN THE THEORY OF LATTICES ${ }^{1}$
}

\section{F. SMILEY}

Introduction. Garrett Birkhoff ${ }^{2}$ derived the following self-dual symmetric condition that a metric lattice be distributive:

$$
\begin{aligned}
2[\mu(a \cup b \cup c)-\mu(a \cap b \cap c)] & =\mu(a \cup b)-\mu(a \cap b)+\mu(a \cup c) \\
& -\mu(a \cap c)+\mu(b \cup c)-\mu(b \cap c) .
\end{aligned}
$$

In a previous note ${ }^{3}$ the author introduced and discussed a generalization of Carathéodory's notion of measurability ${ }^{4}$ with respect to an outer measure function $\mu$ which applies to arbitrary lattices $L$. The $\mu$-measurable elements form a subset $L(\mu)$ consisting of those elements $a \in L$ which satisfy

$$
\mu(a \cup b)+\mu(a \cap b)=\mu(a)+\mu(b)
$$

for every $b \in L$. Closure properties of $L(\mu)$ were investigated. In par-

${ }^{1}$ Presented to the Society, January 1, 1941. The author wishes to express his gratitude to the referee for his valuable suggestions and comments.

${ }^{2}$ Lattice Theory, American Mathematical Society Colloquium Publications, vol. 25 , p. 81 . We shall adopt the notation and terminology of this work and shall indicate specific references to it by $\mathrm{B}$.

${ }^{3} A$ note on measure functions in a lattice, this Bulletin, vol. 46 (1940), pp. 239-241. We shall indicate references to this paper by $\mathrm{M}$.

4 Vorlesungen über Reelle Funktionen, 2d edition, p. 246. 
ticular, $L(\mu)$ was shown to be a sublattice of $L$ when $L$ is modular. It is our purpose here to quantify the relation (1) so as to provide a second (less wide) generalization of measurability. As before we restrict ourselves to modular lattices. A precise strengthening of (2) to yield (1) is first deduced. We then proceed to show that, at least for a positive functional $\mu$, the same closure properties hold as before. We include an examination of the possibility of measurability of complements of measurable elements. Here the influence of distributivity is most apparent; since, under suitable hypotheses, the only measurable elements with "measurable" complements are the measurable neutral elements.

Our two important examples are metric lattices (B, p. 41) and the outer measure of Carathéodory. For the first of these we give, as an application of our method, new proofs of certain explicit and implicit results of $\mathrm{V}$. Gliven $k^{5}{ }^{5}$ concerning relations between distributivity and metric betweenness.

1. Strongly measurable elements. Let us consider a modular lattice $L$ with a least element $O \in L$ and a functional $\mu(a)$ defined on $L$. Note that we do not require that $\mu$ be a modular functional. We define a subset $L_{s}(\mu)$ of strongly $\mu$-measurable elements as those elements $a \in L$ which satisfy (1) for every ${ }^{6} b \in L(\mu)$ and every $c \in L$.

LEMma 1. If an element $a \in L$ is strongly $\mu$-measurable, then it is $\mu$-measurable.

Proof. Take $b=O \in L(\mu)$ in (1) and the result is (2) with $b=c$. A precise relation between strong measurability and distributivity is given in the following theorem.

THeORem 1. An element $a \in L$ is strongly $\mu$-measurable if and only if it is $\mu$-measurable and $\mu(a \cap(b \cup c))=\mu((a \cap b) \cup(a \cap c))$ for every $b \in L(\mu)$ and every $c \in L$.

Proof. We base our proof on the one of Birkhoff (B, p. 81). First let $a \in L_{s}(\mu)$, and consider elements $b \in L(\mu), c \in L$. By Lemma 1, $a \in L(\mu)$; and using (1) and (2) we obtain

5 Contributions à l'étude des systèmes de choses normées, American Journal of Mathematics, vol. 59 (1937), pp. 941-956, and Géométrie des systèmes de choses normées, ibid., vol. 58 (1936), pp. 799-828.

${ }^{6}$ The quantification "for every $b, c \in L$ " would force $O \notin L_{s}(\mu)$ or would make $L_{s}(\mu)$ empty except in the case $L=L(\mu)$. To see this take $a=O$ in (1). Cf. the relations $(\mathrm{a}, \mathrm{b}) \mathrm{D}$ and (a)D of von Neumann's Lectures on Continuous Geometry, Princeton, 1935-1936, p. $38 \mathrm{ff}$. 


$$
\begin{aligned}
2[\mu(a)+\mu(b \cup c)-\mu(a \cap & (b \cup c))-\mu(a \cap b \cap c)] \\
= & \mu(a \cup b)-\mu(a \cap b)+\mu(a \cup c) \\
& -\mu(a \cap c)+\mu(b \cup c)-\mu(b \cap c) .
\end{aligned}
$$

Since $b \in L(\mu)$, expansion of the right member of (3) yields

(4) $2[-\mu(a \cap(b \cup c))-\mu(a \cap b \cap c)]=-2 \mu(a \cap b)-2 \mu(a \cap c)$.

It follows that

$$
\mu(a \cap(b \cup c))=\mu(a \cap b)+\mu(a \cap c)-\mu(a \cap b \cap c) .
$$

Now $L(\mu)$ is a sublattice of $L$ (Theorem 1, M), hence $a \cap b \in L(\mu)$, and we have

$$
\mu(a \cap(b \cup c))=\mu((a \cap b) \cup(a \cap c)) .
$$

To establish the converse, let $a \in L(\mu)$ and consider elements $b \in L(\mu)$, $c \in L$. It is easy to retrace our steps through the equations (6)-(3) and thus obtain (1). This completes the proof.

Remark. By duality we may phrase the criterion of Theorem 1 as $\mu(a \cup(b \cap c))=\mu((a \cup b) \cap(a \cup c))$ for every $b \in L(\mu)$ and every $c \in L$.

Remark. It is known that an element $a$ of a modular lattice is neutral if and only if the correspondences $x \rightarrow x \cup a$ and $x \rightarrow x \cap a$ preserve the lattice operations (B, p. 59). The referee has pointed out the fact that $a \in L_{s}(\mu)$ if and only if these correspondences preserve measurability in the following sense. Define $\mu_{a}^{+}(x) \equiv \mu(a \cup x), \mu_{a}^{-}(x) \equiv \mu(a \cap x)$. Then $a \in L_{s}(\mu)$ if and only if $a \in L(\mu)$ and $y \in L\left(\mu_{a}^{+}\right), y \in L\left(\mu_{a}^{-}\right)$for every $y \in L(\mu)$. To see this let $a \in L_{s}(\mu)$. By Lemma $1, a \in L(\mu)$. Consider an element $y \in L(\mu)$. By Theorem $1(\mathrm{M}), a \cup y \in L(\mu)$ and hence we have for every $x \in L$ that

$$
\mu(a \cup x \cup y)+\mu((a \cup x) \cap(a \cup y))=\mu(a \cup x)+\mu(a \cup y) .
$$

Using the preceding Remark, since $a \in L_{s}(\mu), y \in L(\mu)$, we find that

$$
\mu(a \cup(x \cup y))+\mu(a \cup(x \cap y))=\mu(a \cup x)+\mu(a \cap y) .
$$

This means that $y \in L\left(\mu_{a}^{+}\right)$. Dually $y \in L\left(\mu_{a}^{-}\right)$. Conversely, if $a \in L(\mu)$, $y \in L(\mu)$, and the equation $\left({ }^{*}\right)$ and its dual (which is equation (5)) are true, that is, if $y \in L\left(\mu_{a}^{+}\right)$and $y \in L\left(\mu_{a}^{-}\right)$, then $a \in L_{s}(\mu)$ by the argument used in establishing the converse of Theorem 1.

2. Examples. (1) Let $L_{1}$ be the lattice of all subsets of a set $S$ and $\mu_{1}$ be an outer measure function (in the sense of Carathéodory ${ }^{7}$ ) de-

${ }^{7}$ Op. cit., p. 238. 
fined on $L_{1}$. Here the auxiliary condition of Theorem 1 is automatically satisfied since $L_{1}$ is distributive. Hence $L_{1 s}\left(\mu_{1}\right)=L_{1}\left(\mu_{1}\right)$ in this case. But we have shown (M, p. 239) that $L_{1}\left(\mu_{1}\right)$ consists of the subsets of $S$ measurable (in Carathéodory's sense) with respect to $\mu_{1}$. Thus strong $\mu$-measurability, though more restrictive than $\mu$-measurability, still includes the ordinary idea of measurable set.

(2) Let $L_{2}$ be a metric lattice (in the sense of G. Birkhoff, B, p. 41) and $\mu_{2}$ its modular functional. Then, since $\mu_{2}$ is modular, $L_{2}\left(\mu_{2}\right)=L_{2}$; that is, all elements of $L_{2}$ are $\mu_{2}$-measurable. By Theorem 1, each neutral element of $L_{2}$ is strongly $\mu_{2}$-measurable. Conversely each strongly $\mu_{2}$-measurable element must be neutral. For the criterion of Theorem 1, the fact that $a \cap(b \cup c) \geqq(a \cap b) \cup(a \cap c)$, and the sharply positive character of $\mu_{2}$ imply that $a \cap(b \cup c)=(a \cap b) \cup(a \cap c)$ for every $b, c \in L_{2}$ and dually.

Remark. For our second example the condition (1) holds as an inequality, the left-hand side being smaller. ${ }^{8}$ We see this easily by recalling that (6) holds as an inequality, and by proceeding as in the proof of the converse of Theorem 1 . It is interesting to note also that if a lattice $L$ has an $O$ and an $I$ the condition (1) cannot hold as an inequality (for every $a, c \in L$ and every $b \in L(\mu)$ ) unless $L=L(\mu)$, that is, unless $\mu$ is a modular functional. To see this take $b=O \in L(\mu)$ in (1) and obtain

$$
\mu(a \cup c)+\mu(a \cap c) \leqq \mu(a)+\mu(c) .
$$

Then take $b=I \in L(\mu)$ in (1) and the inequality sign is reversed. Thus (2) holds with $b$ replaced by $c$ for every $a, c \in L$; consequently $\mu$ is a modular functional.

3. Strongly measurable elements a sublattice. The closure of $L_{s}(\mu)$ with respect to the lattice operations $a \cap b$ and $a \cup b$ will be established for positive functionals $\mu$.

THEOREM 2. If $\mu$ is a positive functional, then $L_{s}(\mu)$ is a sublattice of $L$.

Proof. Let $a_{1}, a_{2} \in L_{s}(\mu)$, and consider elements $b \in L(\mu), c \in L$. It clearly suffices by duality, Lemma 1 , Theorem $1(\mathrm{M})$, and Theorem 1 to show that

$$
\mu\left(\left(a_{1} \cap a_{2}\right) \cap(b \cup c)\right)=\mu\left(\left(a_{1} \cap a_{2} \cap b\right) \cup\left(a_{1} \cap a_{2} \cap c\right)\right) .
$$

We always have $x \equiv\left(a_{1} \cap a_{2}\right) \cap(b \cup c) \geqq\left(a_{1} \cap a_{2} \cap b\right) \cup\left(a_{1} \cap a_{2} \cap c\right) \equiv y$, and hence $\mu(x) \geqq \mu(y)$. The reverse inequality may be proved thus:

${ }^{8}$ This was noted by the referee. 


$$
\begin{aligned}
\mu(x) & =\mu\left(a_{1}\right)+\mu\left(a_{2} \cap(b \cup c)\right)-\mu\left(a_{1} \cup\left(a_{2} \cap(b \cup c)\right)\right) \\
& =\mu\left(a_{1}\right)+\mu\left(\left(a_{2} \cap b\right) \cup\left(a_{2} \cap c\right)\right)-\mu\left(a_{1} \cup\left(a_{2} \cap(b \cup c)\right)\right) \\
& \leqq \mu\left(a_{1}\right)+\mu\left(\left(a_{2} \cap b\right) \cup\left(a_{2} \cap c\right)\right)-\mu\left(a_{1} \cup\left(a_{2} \cap b\right) \cup\left(a_{2} \cap c\right)\right) \\
& =\mu\left(a_{1} \cap\left(\left(a_{2} \cap b\right) \cup\left(a_{2} \cap c\right)\right)\right)=\mu(y) .
\end{aligned}
$$

The proof is complete. The author has been unable to decide the question of whether Theorem 2 remains true when the hypothesis that $\mu$ be positive is suppressed.

Let $N(L) \subset L$ denote the sublattice of neutral elements of $L$. Then we have $L(\mu) \supset L_{s}(\mu) \supset N(L) \cdot L(\mu)$. Examples will be given where each of these inequalities is strict. For the first, Example (2) of $\$ 2$ will suffice; while a simple example, to be given in $\$ 5$, settles the second.

4. Monotone sequences of elements of $L_{s}(\mu)$. The closure properties of $L_{s}(\mu)$ with respect to $(o)$-limits of monotone denumerable sequences can easily be related (as for $L(\mu)$ ) to continuity properties of the functional $\mu$. We first restate the following definition (cf. M).

DEFINITION. If for each sequence $a_{i} \uparrow a\left(a_{i} \downarrow a\right)$ of elements of $L(\mu)$ we have $\mu\left(a_{i} \cup b\right) \rightarrow \mu(a \cup b)$ and $\mu\left(a_{i} \cap b\right) \rightarrow \mu(a \cap b)$ for every $b \in L$, we say that $(L, \mu)$ satisfies $B^{+}\left(B^{-}\right)$.

THEOREM 3. If the functional $\mu$ is positive, then a sufficient condition that (o)-limits of increasing (decreasing) sequences of elements of $L_{s}(\mu)$ which belong to $L$ also belong to $L_{s}(\mu)$ is that $(L, \mu)$ satisfy $B^{+}\left(B^{-}\right)$.

Proof. Let $(L, \mu)$ satisfy $B^{+}$, and consider an increasing sequence of elements of $L_{s}(\mu): a_{i} \uparrow a \in L$. By Lemma 1 and Theorem 2 (M) we know that $a \in L(\mu)$. For every $b \in L(\mu)$ and every $c \in L$ we have, by Theorem 1, that

$$
\begin{aligned}
\mu\left(a_{i} \cap(b \cup c)\right) & =\mu\left(\left(a_{i} \cap b\right) \cup\left(a_{i} \cap c\right)\right) \\
& =\mu\left(a_{i} \cap b\right)+\mu\left(a_{i} \cap c\right)-\mu\left(a_{i} \cap b \cap c\right) .
\end{aligned}
$$

On taking the limit as $i \rightarrow \infty$ and using $B^{+}$we get

$$
\begin{aligned}
\mu(a \cap(b \cup c)) & =\mu(a \cap b)+\mu(a \cap c)-\mu(a \cap b \cap c) \\
& =\mu((a \cap b) \cup(a \cap c))
\end{aligned}
$$

for every $b \in L(\mu)$ and every $c \in L$. Thus $a \in L_{s}(\mu)$ by Theorem 1 . The alternate reading is dual. The proof is complete. It is easy to formulate a sufficient condition on $(L, \mu)$ that the condition of Theorem 3 be necessary as well as sufficient.

5. Measurability of complements. In our Examples (1) and (2) of 
$\$ 2$ it is known that complements of strongly $\mu$-measurable elements are unique and strongly $\mu$-measurable. ${ }^{9}$ This is not true in general. In fact, complements of strongly $\mu$-measurable elements may fail to be even $\mu$-measurable. To see this, consider the simplest modular, non-distributive lattice $L_{0}$ of five elements $O, a, b, c, I$, with $O$ and $I$ least and greatest elements, respectively. Define $\mu(O)=0, \mu(I)=\frac{5}{6}$, $\mu(a)=\frac{1}{3}$, and $\mu(b)=\mu(c)=\frac{1}{2}$. It is easily seen that $a \in L_{0 s}(\mu)$. However, it is obvious that neither $b$ nor $c$ (each a complement of $a$ ) belongs to $L_{0}(\mu)$. In this direction we may prove the following theorem.

THEOREM 4. If $\mu$ is sharply positive, then a complement of a strongly $\mu$-measurable element is $\mu$-measurable if and only if it is unique, and then it is itself strongly $\mu$-measurable.

Proof. Consider an element $a \in L_{s}(\mu)$ with a complement $x \in L(\mu)$. Suppose $y$ is a second complement of $a$. By Theorem 1,

$$
\mu(a \cap(x \cup y))=\mu((a \cap x) \cup(a \cap y))=\mu(O) .
$$

Since $\mu$ is sharply positive $a \cap(x \cup y)=O$, and it follows that $x \cup y$ is a complement of $a$. But every complement $z$ of $a$ gives $\mu$ the same value; since, by (2),

$$
\mu(O)+\mu(I)=\mu(a)+\mu(z) .
$$

Thus $\mu(x \cup y)=\mu(x)=\mu(y)$, and consequently, since $\mu$ is sharply positive, $x \cup y=x=y$. Hence $x$ is unique. Conversely, let $a^{\prime}$ be the unique complement of $a$. Then by Theorem 4.5 (B), $a$ and $a^{\prime}$ are neutral. To show that $a^{\prime} \in L(\mu)$ (and hence that $a^{\prime} \in L_{s}(\mu)$ ), consider an element $b \in L$. Since, by Lemma $1, a \in L(\mu)$, we have

$$
\begin{aligned}
\mu(a)+\mu\left(a^{\prime} \cap b\right) & =\mu\left(a \cup\left(a^{\prime} \cap b\right)\right)+\mu\left(a \cap a^{\prime} \cap b\right), \\
& =\mu\left(\left(a \cup a^{\prime}\right) \cap(a \cup b)\right)+\mu(O), \\
& =\mu(a \cup b)+\mu(O) .
\end{aligned}
$$

Dually,

$$
\mu(a)+\mu\left(a^{\prime} \cup b\right)=\mu(a \cap b)+\mu(I) .
$$

Adding equations (8) and (9), using (2) and (7), we find that

$$
2 \mu(a)+\mu\left(a^{\prime} \cup b\right)+\mu\left(a^{\prime} \cap b\right)=2 \mu(a)+\mu\left(a^{\prime}\right)+\mu(b) .
$$

This gives (2) with $a=a^{\prime}$ at once. The proof is complete. We note

${ }^{9}$ For example (1), see Carathéodory, op. cit., p. 248; while Birkhoff's Theorem 4.5 (B, p. 59) covers Example (2); or see the remark which closes this section. 
that no use was made of the sharply positive character of the functional $\mu$ in establishing the converse of this theorem.

Remark. The converse of Theorem 4 shows that the complements of strongly $\mu$-measurable elements will be strongly $\mu$-measurable (and unique) whenever $L_{s}(\mu)$ consists entirely of neutral elements. This "explains" why this closure property holds in our Examples (1) and (2)..$^{10}$

6. Relation to certain work of V. Glivenko. Let us now consider in more detail our Example (2) of \$2. V. Glivenko obtained, among other results, a characterization of elements of the center (the complemented neutral elements) of $L_{2}$ in terms of metric betweenness properties. ${ }^{11}$ Examination of his work will show that the neutral elements themselves may be similarly characterized in the following way.

LeMma 2. An element $u$ of a metric lattice $L$ is neutral if and only if $(a \cap b) \cup(u \cap(a \cup b))$ is between $a$ and $b$ for every $a, b \in L$.

Proof. We give a separate proof based on the condition (1). Consider an element $u \in L$ for which the condition holds. For every $a$, $b \in L$ we have, setting $x=(a \cap b) \cup(u \cap(a \cup b)), \delta(a, x)+\delta(x, b)$ $=\delta(a, b)$, which becomes, in terms of $\mu$,

$$
\begin{array}{r}
\mu(a \cup(u \cap(a \cup b)))-\mu((a \cap b) \cup(a \cap u))+\mu(b \cup(u \cap(a \cup b))) \\
-\mu((a \cap b) \cup(b \cap u))=\delta(a, b) .
\end{array}
$$

Expansion of the left member gives

$$
\begin{array}{r}
\mu(a)+\mu(b)+2 \mu(u \cap(a \cup b))-2 \mu(a \cap u)-2 \mu(b \cap u)-2 \mu(a \cap b) \\
+2 \mu(a \cap b \cap u)=\delta(a, b) .
\end{array}
$$

Further expansion shows that

$$
\begin{aligned}
\mu(a)+\mu(b)- & 2 \mu(u \cup a \cup b)+2 \mu(u)+2 \mu(a \cup b)-2 \mu(a \cap b) \\
- & 2 \mu(a \cap u)-2 \mu(b \cap u)+2 \mu(u \cap a \cap b)=\delta(a, b) .
\end{aligned}
$$

Transposing, we have

$$
\begin{aligned}
\delta(a, b)+\mu(a)+\mu(u)-2 \mu(a \cap u)+\mu(b) & +\mu(u)-2 \mu(b \cap u) \\
& =2 \delta(u \cup a \cup b, u \cap a \cap b) .
\end{aligned}
$$

\footnotetext{
${ }^{10}$ It would be desirable, as the referee has pointed out, to construct an abstract theory which more completely unites our two examples. Our attempts in this direction have so far been unsatisfactory.

11 Primus op. cit., p. 947. In a metric space, the point $q$ is said to be between the point $p$ and $r$ in case $\delta(p, q)+\delta(p, r)=\delta(p, r)$. The reader is referred to L. M. Blumenthal's book Distance Geometries for a discussion of this relation.
} 
Thus, finally,

$$
\delta(a, b)+\delta(b, u)+\delta(u, a)=2 \delta(u \cup a \cup b, u \cap a \cap b),
$$

which is merely the condition (1) written in terms of the metric $\delta$. Therefore $u$ is neutral. The converse may be proved by reversing the steps of our argument. The proof is complete.

We note that an element $v$ is a complement of $u$ if and only if both $O$ and $I$ are between $u$ and $v$.

For brevity's sake let us write $(a, b, c)$ for the expression, "the element $b$ is between the elements $a$ and $c$. " Glivenko calls a betweenness relation transitive ${ }^{12}$ if the relations $(x, c, y),(a, x, b)$ and $(a, y, b) \mathrm{im}$ ply the relation $(a, c, b)$. He proves the facts of our final theorem.

TheOREM 5. An element $u$ of a metric lattice $L$ is neutral if and only if either (i) $a \cap b \leqq u \leqq a \cup b$ implies that $(a, u, b)$ for every $a, b \in L$, or (ii) metric betweenness is transitive in $L$.

Proof. Using the condition (1) we prove the first of these results in the following way. Suppose $a \cap b \leqq u \leqq a \cup b$, then

$$
\begin{aligned}
\delta(a, b)=\mu(a \cup b)-\mu(a \cap b) & =\mu(a \cup b \cup u)-\mu(a \cap b \cap u) \\
& =\delta(a \cup b \cup u, a \cap b \cap u) .
\end{aligned}
$$

Hence, if $(a, u, b)$, then

$$
2 \delta(a \cup b \cup u, a \cap b \cap u)=2 \delta(a, b)=\delta(a, b)+\delta(a, u)+\delta(u, b) ;
$$

which is (1). Conversely, if (1) is valid and $a \cap b \leqq u \leqq a \cup b$, then $\delta(a, b)=\delta(a, u)+\delta(u, b)$, that is, $(a, u, b)$. This proves the first result. The second follows easily from Lemma 2 and the first. For, let metric betweenness be transitive and consider elements $u, a, b \in L$. Take $x=a \cap b, y=a \cup b, c=(a \cap b) \cup(u \cap(a \cup b))$. We then have $(x, u, y)$ since $x \leqq u \leqq y$; and by the modularity of $\mu$ we know that $(a, x, b)$ and that $(a, y, b)$. Transitivity of metric betweenness implies that $(a \cap b) \cup(u \cap(a \cup b))$ is between $a$ and $b$ for every $a, b \in L$. It follows from Lemma 2 that $u$ is neutral. We can now follow Glivenko in a proof of the converse. If $(x, u, y),(a, x, b)$, and $(a, y, b)$, then $x \cap y \leqq u \leqq x \cup y, a \cap b \leqq x \leqq a \cup b, a \cap b \leqq y \leqq a \cup b$, and it is easily seen that $a \cap b \leqq u \leqq a \cup b$. If $u$ is neutral we then obtain that $(a, u, b)$ from the first result; and metric betweenness is transitive in $L$.

\section{LEHIGH UNIVERSITY}

12 Secundus op. cit. For further transitivities of this nature and their application to a definition of betweenness in general lattices, see Transitivities of betweenness by Everett Pitcher and the author. 